\title{
GENERIC MORSE-SMALE DIFFEOMORPHISMS HAVE ONLY TRIVIAL SYMMETRIES
}

\author{
YOSHIO TOGAWA
}

\begin{abstract}
The purpose of this paper is to prove that for a $C^{1}$-generic Morse-Smale diffeomorphism $f$, the set of symmetries of $f, Z(f)$, is equal to $\left\{f^{k} \mid k \in \mathbf{Z}\right\}$.
\end{abstract}

1. Introduction. Let $M$ be a compact connected $C^{\infty}$-manifold without boundary. Let $\operatorname{Diff}(M)$ be the set of $C^{1}$-diffeomomrphisms of $M$ with $C^{1}$-topology. Let MS denote the open set of all Morse-Smale diffeomorphisms of $M$ in $\operatorname{Diff}(M)$ [5]. For $f \in \operatorname{Diff}(M)$ we say $g \in \operatorname{Diff}(M)$ is $a$ symmetry of $f$ iff $f \circ g=g \circ f$. Then the centralizer $Z(f)$ of $f$ is the set of all symmetries of $f$. Clearly, $f^{k}$ is a symmetry of $f$ for any $k \in \mathbf{Z}(\mathbf{Z}$ is the set of integers). We call such symmetries trivial symmetries. A proper symmetry is a symmetry which is not trivial. The following question is posed by N. Kopell [4] and J. Palis [6].

Is the set of diffeomorphisms without proper symmetry generic in $\operatorname{Diff}(M)$ ?

In this paper, we shall prove the following theorem which gives an affirmative solution of the conjecture in MS.

THEOREM. It is $C^{1}$-generic in MS that $f$ has no proper symmetry.

The referee pointed out that the work of Boyd Anderson in [1] is closely related to ours. See also [2].

I would like to thank Professor Hiroshi Noguchi for his kind advice. I would also like to thank the referee for pointing out how to overcome an error in the proof of Lemma 5.1.

2. Proof of the Theorem. Choose a riemannian metric on $M$. For a tangent vector $v$ we let $\|v\|$ denote the length of $v$ by this riemannian metric. Let $J^{1}(M)$ denote the 1-jet space on $M\left(=\cup_{x, y \in M} L\left(T_{x} M, T_{y} M\right)\right)$, and $\pi_{i}$ : $J^{1}(M) \rightarrow M(i=1,2)$ denote the projections, i.e., $\pi_{1}(\alpha)=x, \pi_{2}(\alpha)=y$ for $\alpha \in L\left(T_{x} M, T_{y} M\right)$. For $m \in \mathbf{N}$ ( $\mathbf{N}$ is the set of natural numbers) we define $J^{1}(M: m)$ as the set of all $\alpha^{\prime}$ 's in $J^{1}(M)$ such that $1 / m \leqslant\|\alpha v\| \leqslant m$, for any tangent vector $v$ of norm 1 . We fix a countable basis $\Theta$ of the topology of $M$ and a countable dense subset $M^{*}$ of $M$. Let

Received by the editors July 2, 1976 and, in revised form, December 3, 1976.

AMS (MOS) subject classifications (1970). Primary 58F99.

Key words and phrases. Morse-Smale diffeomorphism, symmetry, centralizer, trivial symmetry, proper symmetry. 


$$
\Gamma=\left\{\left(U, x_{0}, m\right) \mid U \in \Theta, x_{0} \in M^{*}, x_{0} \in U, m \in \mathbf{N}\right\} .
$$

Definition 2.1. Let $\left\{B\left(U, x_{0}, m\right)\right\}_{\Gamma}$ be the family defined as follows; for $\left(U, x_{0}, m\right) \in \Gamma, B\left(U, x_{0}, m\right)$ is the set of all Morse-Smale diffeomorphism $f$ 's which have a mapping $S: O_{f}\left(x_{0}\right) \rightarrow J^{1}(M: m)$ satisfying the following conditions;

(i) $\pi_{1} \circ S=$ identity,

(ii) $\pi_{2} \circ S\left(x_{0}\right) \notin O_{f}(U)$ and

(iii) $T f \circ S(x)=S(f(x)) \circ T_{x} f$, for any $x$ in $O_{f}\left(x_{0}\right)$, where $O_{f}\left(x_{0}\right)$ denotes the orbit of $x_{0}$.

REMARK. Condition (iii) implies that

$$
f\left(\pi_{2} \circ S(x)\right)=\pi_{2} \circ S(f(x))
$$

for any $x \in O_{f}\left(x_{0}\right)$. Therefore (ii) implies that

(ii) $\pi_{2} \circ S(x) \notin O_{f}(U)$ for any $x \in O_{f}\left(x_{0}\right)$.

In order to prove the Theorem it is sufficient to verify Propositions 2.1, 2.2 and 2.3.

Proposition 2.1. If $f \in \mathrm{MS}$ has a proper symmetry, then $f$ is contained in one of $B\left(U, x_{0}, m\right)$ 's.

Proposition 2.2. Each $B\left(U, x_{0}, m\right)$ is closed.

Proposition 2.3. Each $B\left(U, x_{0}, m\right)$ has no interior point.

In the following sections, we shall prove these propositions.

3. Proof of Proposition 2.1. We need the following lemma:

Lemma 3.1. Let $f \in \mathrm{MS}$ and $g \in \operatorname{Diff}(M)$. Suppose that $g(x) \in O_{f}(x)$ for any $x \in M^{*}-\operatorname{per} f$. Then $g$ is a trivial symmetry of $f$.

Proof. Let $A_{k}=\left\{x \in M-\operatorname{per} f \mid g(x)=f^{k}(x)\right\}$. Then the family $\left\{A_{k}\right\}_{k \in \mathbf{Z}}$ is disjoint, each $A_{k}$ is closed in $M-\operatorname{per} f$, and $M^{*}-\operatorname{per} f$ is contained in $\cup A_{k}$. Let $V$ be a connected open set such that $\mathrm{Cl}(V) \subset M-$ per $f$. Since $V$ is open, $V \cap M^{*}$ is dense in $\mathrm{Cl}(V)$. We claim that $\mathrm{Cl}(V) \subset A_{k}$ for some $k \in \mathbf{Z}$. Let $Q_{\varepsilon}(x)=\{y \in M \mid d(x, y)<\varepsilon\}$. Since $\mathrm{Cl}(V)$ consists only of wandering points, there exists $\varepsilon>0$ such that $f^{n}\left(Q_{\varepsilon}(x)\right) \cap f^{n^{\prime}}\left(Q_{\varepsilon}(x)\right)$ $=\varnothing$ for any distinct integers $n$ and $n^{\prime}$, and any $x \in \mathrm{Cl}(V)$ because of the compactness of $\mathrm{Cl}(V)$. Then there exists $\varepsilon^{\prime}>0$ such that $g\left(Q_{\varepsilon^{\prime}}(x)\right) \subset$ $f^{k}\left(Q_{e}(x)\right)$ for any $x \in A_{k} \cap V$. Notice that $\varepsilon^{\prime}$ depends on $k$ but this presents no problem. Let $x^{*} \in A_{k} \cap V \cap M^{*}$. Since $g\left(Q_{\varepsilon^{\prime}}\left(x^{*}\right)\right)$ is contained in $f^{k}\left(Q_{e}\left(x^{*}\right)\right)$ and $M^{*} \cap V \subset \cup A_{k}, Q_{\varepsilon^{\prime}}\left(x^{*}\right) \cap M^{*}$ is contained in $A_{k}$. This implies that $Q_{\varepsilon^{\prime}}\left(x^{*}\right) \subset A_{k}$, and since $V$ is connected, $V$ is contained in $A_{k}$.

Let $B_{1}, \ldots, B_{s}$ denote the connected components of $M-\operatorname{per} f$. We can choose a connected open set $V$ for any points $x$ and $y$ in $B_{i}$ such that $x, y \in \mathrm{Cl}(V) \subset B_{i}$, so $B_{i}$ is contained in $A_{k}$ for some $k \in \mathbf{Z}$.

If $\operatorname{dim}(M) \geqslant 2$, then $M-\operatorname{per} f$ is connected, and equivalently, $M-\operatorname{per} f$ $=B_{1}$; hence $g$ is trivial. 
If $\operatorname{dim}(M)=1$, equivalently, $M=S^{1}, g$ can be nontrivial only if there is a periodic point $p$ such that $g=f^{k}$ in the right neighbourhood of $p$ and $g=f^{k^{\prime}}$ in the left neighbourhood of $p$. But this contradicts with the assumption that $p$ is hyperbolic. Hence $g$ is trivial.

Proof of Proposition 2.1. Let $f$ be a Morse-Smale diffeomorphism with a proper symmetry $g$. By Lemma 3.1 , we can choose a point $x_{0} \in M^{*}-\operatorname{per} f$ such that $g\left(x_{0}\right) \notin O_{f}\left(x_{0}\right)$. We show that we can choose $U \in \Theta$ such that $x_{0} \in U$ and $O_{f}\left(g\left(x_{0}\right)\right) \cap O_{f}(U)=\varnothing$. Since $x_{0} \notin$ nonwandering set, it is not in either $\alpha$ - or $\omega$-limit set of $g\left(x_{0}\right)$; since it is by hypothesis not in $O_{f}\left(g\left(x_{0}\right)\right)$, we can conclude that the point $x_{0}$ does not belong to the closed set $\mathrm{Cl}\left(O_{f}\left(g\left(x_{0}\right)\right)\right)$, so there exists a neighbourhood $U \in \Theta$ of $x_{0}$ disjoint from $O_{f}\left(g\left(x_{0}\right)\right)$; but then $O_{f}(U) \cap O_{f}\left(g\left(x_{0}\right)\right)=\varnothing$. Let us choose $m \in \mathbf{N}$ such that $m>\max \left(\|T g v\|,\left\|T g^{-1} v\right\|\right)$ for any $v \in T M$ of norm 1. Define $S(x)$ by $S(x)=T_{x} g$ for $x \in O_{f}\left(x_{0}\right)$. It is clear that $f \in B\left(U, x_{0}, m\right)$.

4. Proof of Proposition 2.2. Suppose that a sequence $\left\{f_{n}\right\}$ of diffeomorphisms of $B\left(U, x_{0}, m\right)$ converges to $f \in \operatorname{Diff}(M)$. For each $f_{n}$ we choose a map $S_{n}$ which satisfies the conditions of Definition 2.1. Let us define a map $S$ for $f$ as follows. Since $J^{1}(M: m)$ is compact, the sequence $S_{n}\left(x_{0}\right)$ has cluster points. Define $S\left(x_{0}\right)$ to be one of the cluster points. Then $S\left(x_{0}\right) \in J^{1}(M: m)$ and $\pi_{1} \circ S\left(x_{0}\right)=x_{0}$. We define

$$
S: O_{f}\left(x_{0}\right) \rightarrow J^{1}(M: m)
$$

by

$$
S\left(x_{k}\right)=T f^{k} \circ S\left(x_{0}\right) \circ T f^{-k} \mid T_{x_{k}} M
$$

for $x_{k}=f^{k}\left(x_{0}\right)$, where $T_{x_{k}} M$ denotes the tangent plane on $x_{k}$. Clearly $S$ satisfies conditons (i) and (iii) of Definition 2.1. We check that $S$ satisfies condition (ii). First notice that

$$
f^{k}\left(\pi_{2} \circ S\left(x_{0}\right)\right) \subset \mathrm{Cl}\left(\left\{f_{n}^{k}\left(\pi_{2} \circ S_{n}\left(x_{0}\right)\right)\right\}_{n \in \mathrm{N}}\right)
$$

since $\pi_{2} \circ S\left(x_{0}\right)$ is a cluster point of $\left\{\pi_{2} \circ S_{n}\left(x_{0}\right)\right\}_{n \in N}$ and $\left\{f_{n}^{k}\right\}_{n \in N}$ converges to $f^{k}$ for any fixed $k$. But since any $f_{n}^{k}\left(\pi_{2} \circ S_{n}\left(x_{0}\right)\right)$ is not in $U$, neither is $f^{k}\left(\pi_{2} \circ S\left(x_{0}\right)\right)$ in $U$; this implies condition (ii) of Definition 2.1.

5. Proof of Proposition 2.3. Let $\rho: \mathbf{R}^{q} \rightarrow \mathbf{R}^{q}$ be a $C^{\infty}$-function with the following properties:

(i) $\max (\|\rho\|,\|D \rho\|) \leqslant 1$,

(ii) $\rho(0)=0$ and $D \rho(0)=$ identity, and

(iii) $\rho(x)=0$ for any $\|x\| \geqslant 1$.

Definition 5.1. A number sequence $\left\{a_{n}\right\} \rightarrow 0$ is called of exponential type iff for some $a>0$ and $K>0, a^{n} / K \leqslant a_{n} \leqslant K a^{n}$ for any $n \in \mathbf{N}$.

LEMMA 5.1. Let L: $\mathbf{R}^{q} \rightarrow \mathbf{R}^{q}$ be a semisimple linear contraction, i.e., L has a matrix $\left(a_{i j}\right)$ such that 


$$
\begin{aligned}
a_{2 i-1,2 i-1} & =\left|\lambda_{i}\right| \cos \theta_{i}, & a_{2 i-1,2 i} & =-\left|\lambda_{i}\right| \sin \theta_{i}, \\
a_{2 i, 2 i-1} & =\left|\lambda_{i}\right| \sin \theta_{i}, & a_{2 i, 2 i} & =\left|\lambda_{i}\right| \cos \theta_{i},
\end{aligned}
$$

for $1 \leqslant i \leqslant q^{\prime}$, and $a_{i i}=\lambda_{i}$ for $2 q^{\prime} \leqslant i$, and the others $=0$ for some $0 \leqslant q^{\prime} \leqslant$ $q / 2$ and $0<\left|\lambda_{i}\right|<1$.

Let $B=\left\{x \in \mathbf{R}^{q} \mid\|x\|<1\right\}$ and $e_{1}=(1,0, \ldots, 0)$. Suppose that $0 \neq x_{0} \in$ $B$ and let $U \in B$ be an open neighbourhood of $x_{0}$ stich that $L^{n}(U) \cap L^{n^{\prime}}(U)=$ $\varnothing$ for any distinct integers $n$ and $n^{\prime}$. Then for any $\varepsilon>0$ there is a $C^{1}$-local diffeomorphism $f: B \rightarrow \mathbf{R}^{q}$ such that

(i) $\operatorname{Max}(\|f-L\|,\|D f-D L\|)<\varepsilon$,

(ii) $f\left|B-O_{f}(U)=L\right| B-O_{L}(U)$,

(iii) the sequence $\left\{\left\|D f^{n}\left(x_{0}\right) e_{1}\right\|\right\}$ is not of exponential type.

Proof. Let $x_{n}=L^{n} X_{0}$. We ghoose $\delta(n)$ such that $0<\delta(n)<$ $\min \left(\left\|x_{n}\right\| / 2, d\left(x_{n}, B-L^{n}(U)\right)\right)$ for $h \in \mathbf{N}$. Define $\rho_{n}$ by

$$
\rho_{n}(x)=\varepsilon \delta(n) \rho(x / \delta(n)) \text {. }
$$

We define $f$ by

$$
f\left|B-O_{L}(U)=L\right| B-O_{L}(U)
$$

and

$$
f(x)=L x+\rho_{n+1}\left(L\left(x-x_{n}\right)\right) /(n+1)
$$

for $x \in L^{n}(U)$. Then $f$ is well defined as a continuous mapping and of class $C^{1}$ on $B-\{0\}$. Since

$$
\|D f(x)-L\| \leqslant\left\|D \rho_{n+1}\right\| \cdot\|L\| /(n+1)<\varepsilon /(n+1)
$$

for $x \in L^{n}(U)$, then $D f(x) \rightarrow L$ as $x \rightarrow 0$, and hence $f$ is continuously differentiable at 0 . Clearly $C^{1}$-distance between $f$ and $L$ is less than $\varepsilon$, so $f$ is a diffeomorphism provided that $\varepsilon$ is sufficiently small.

Now we check that the convergence of the sequence $\left\|D f^{n}\left(x_{0}\right) e_{1}\right\|$ is not of exponential type. Let $e_{1}^{n}=D f^{n}\left(x_{0}\right) e_{1}$. Then

$$
\left\|e_{1}^{n+1}\right\|=\left\|D f\left(x_{n}\right) e_{1}^{n}\right\|=\left(\left|\lambda_{1}\right|+\left|\lambda_{1}\right| \varepsilon /(n+1)\right)\left\|e_{1}^{n}\right\|
$$

and

$$
\left\|D f^{n}\left(x_{0}\right) e_{1}\right\|=\left\|e_{1}^{n}\right\|=\prod_{i=1}^{i=n}\left|\lambda_{1}\right|(1+\varepsilon / i) .
$$

Notice that the convergence of the sequence $\left\{\left\|e_{1}^{n}\right\|\right\}$ is of exponential type iff the sequence $\prod_{i=1}^{i=n}(1+\varepsilon / i)$ converges. But since a sequence $\Pi\left(1+a_{n}\right)$ converges iff $\Sigma a_{n}$ does [4, Theorem 3, p. 94], the sequence $\left\{\left\|e_{1}^{n}\right\|\right\}$ is not of exponential type.

Proof of Proposition 2.3. Let $f \in B\left(U, x_{0}, m\right)$. We approximate $f$ by a Morse-Smale diffeomorphism which is not in $B\left(U, x_{0}, m\right)$. We first approximate $f$ by $f_{1}$ which has the following properties:

(i) there exists a sink $p$ of $f_{1}$ such that $x_{0} \in W^{s}(p)$,

(ii) for any periodic point $q$ of $f_{1}$, setting $l(q)$ equal to the period of $q$ under 
$f_{1}, f_{1}^{l(q)}$ is semisimple linear in some chart.

Since $B\left(U, x_{0}, m\right) \subset B\left(U^{\prime}, x_{0}, m\right)$ for any neighbourhood $U^{\prime}$ of $x_{0}$ such that $U^{\prime} \in \Theta$ and $U^{\prime} \subset U$, we can suppose, without loss of generality, $U \subset$ $W^{s}(p)$ and $f_{1}^{n}(U) \cap f_{1}^{n^{\prime}}(U)=\varnothing$ for any distinct integers $n$ and $n^{\prime}$. By (ii), if $\left\{\left\|T f_{1}^{n} v\right\|\right\}$ converges to 0 , then the convergence is of exponential type for any nonzero tangent vector $v$. Let us perturb $f_{1}$ near $p$, as in Lemma 5.1, by taking $f_{1}$ as the linear map $L$. Then we get an approximation $f_{2}$ of $f_{1}$ with a tangent vector $v$ on $x_{0}$ such that the sequence $\left\{\left\|T f_{2}^{n} v\right\|\right\}$ is not of exponential type. Then we show that $f_{2} \notin B\left(U, x_{0}, m\right)$. Suppose the contrary, and consider the sequence

$$
\left\{\left\|S\left(x_{n}\right) T f_{2}^{n} v\right\|\right\}=\left\{\left\|T f_{2}^{n} S\left(x_{0}\right) v\right\|\right\}
$$

where $S$ is a map for $f_{2}$ in Definition 2.1. Since $S\left(x_{0}\right) v$ is a tangent vector on $M-O_{f_{2}}(U)$ and $f_{2}$ coincides with $f_{1}$ on $O_{f_{1}}(U)=O_{f_{2}}(U)$, the sequence $\left\{\left\|S\left(x_{n}\right) T f_{2}^{n} v\right\|\right\}=\left\{\left\|T f_{2}^{n} S\left(x_{0}\right) v\right\|\right\}$ is of exponential type. Since

$$
\left\|T f_{2}^{n} v\right\| / m \leqslant\left\|S\left(x_{n}\right) T f_{2}^{n} v\right\| \leqslant m\left\|T f_{2}^{n} v\right\|
$$

by Definition 2.1, so

$$
\left\|S\left(x_{n}\right) T f_{2}^{n} v\right\| / m \leqslant\left\|T f_{2}^{n} v\right\| \leqslant m\left\|S\left(x_{n}\right) T f_{2}^{n} v\right\| .
$$

But $\left\{\left\|S\left(x_{n}\right) T f_{2}^{n} v\right\|\right\}$ is of exponential type; then $\left\{\left\|T f_{2}^{n} v\right\|\right\}$ is also of exponential type, a contradiction.

\section{REFERENCES}

1. B. Anderson, Centralizers of $C^{\infty}$-diffeomorphisms, Trans. Amer. Math. 222 (1976), 97-106.

2. __ Diffeomorphisms with discrete centralizer, Topology 15 (1976), 143-147.

3. K. Knopp, Infinite sequences and series, Dover, New York, 1956.

4. N. Kopell, Commuting diffeomorphisms, Sympos. on Differential Equations and Dynamical Systems, Lecture Notes in Math., Vol. 204, Springer-Verlag, Berlin and New York, 1971.

5. J. Palis, On Morse-Smale dynamical systems, Topology 8 (1969), 385-404.

6. __ Vector fields generate few diffeomorphisms, Bull. Amer. Math. Soc. 80 (1974), $503-505$.

Department of Mathematics, School of Science and Engneernag, Wasta chivemetry, SHINJUKU, TOKYO, JAPAN

Current address: Science University of Tokyo, Department of Information Science, Faculty of Science and Technology, Noda City (278), Japan 